\title{
Is the Neutrophil-to-Lymphocyte Ratio more correlated than C-reactive protein with postoperative complications after major abdominal surgery?
}

Patrice Forget, Valerie Dinant, Marc De Kock

Background: The Neutrophil-to-Lymphocyte Ratio (NLR) is an inflammatory marker that has proven usefulness for predicting late complications. Whether it is associated with immediate postoperative complications after abdominal surgery is not known. In this study, we attempted to correlate the NLR and the C-reactive protein (CRP) with postoperative complications rate. Methods: We performed a post-hoc analysis of previously collected data concerning 82 consecutive patients (median age: 62 years, range: $27-80$, female/male $32 / 50$ ) undergoing major abdominal surgeries. For each patient, we recorded preoperative characteristics, the NLR and CRP values, and postoperative complications (between $D+8$ and $D+30)$ such as infections $(N=29)$, cardiovascular complications $(\mathrm{N}=12)$ and other complications $(\mathrm{N}=28)$. We performed uni- and multivariate analyses using logistic/linear regression models. Results: Patients with complications did not present a higher preoperative NLR than those without, but a higher ratio at $D+7$ (10.73 \pm 9.86 vs. $4.73 \pm 3.38$ without complication) $(P<0.001)$. In the univariate analysis, the NLR at $D+7$ was associated with postoperative complications $(P<0.001)$. At $D+7$, in the multivariate analysis, an increased NLR was associated with more complications $(P<0.001)$, whereas none of the other factors, including CRP, showed any correlation. Conclusion: Postoperative NLR at day 7 after major abdominal surgery is associated with complications during the first postsurgical month, in contrast with the CRP level. The NLR is a simple and interesting parameter in the perioperative period. 
1 Title: Is the Neutrophil-to-Lymphocyte Ratio more correlated than C-reactive protein with

2 postoperative complications after major abdominal surgery?

4 Authors: FORGET Patrice, M.D. Ph.D., DINANT Valérie, M.D., DE KOCK Marc, M.D, Ph.D.

5 Name of Department(s) and Institution(s): Department of Anesthesiology; Cliniques

6 universitaires Saint-Luc; Institute of Neuroscience; Université catholique de Louvain, Brussels,

7 Belgium.

8 Corresponding Author and Reprints: Patrice Forget, Departments of Anesthesiology, St-Luc

9 Hospital, av. Hippocrate 10-1821, 1200 Brussels, Belgium. Telephone number +3227641821 .

10 Address e-mail to forgetpatrice@yahoo.fr

11 Financial Support: This work was exclusively supported by the Department of Anesthesiology

12 of the Cliniques universitaires Saint-Luc, Brussels, Belgium.

13

14

15 Keywords: Neutrophil-to-lymphocyte ratio, C-reactive protein, postoperative complications 
Introduction

21 Surgery induces an acute inflammatory response and the early postoperative period can be

22 place of many complications. Despite advances in surgical techniques and perioperative

23 medicine, excessive surgical stress response can be associated, and even lead, to serious post-

24 operative complications, like surgical site infection, sepsis and multiple organ failure. ${ }^{1}$ This

25 response can be reflected, at least partially, by biomarkers that may help the clinician to monitor

26 the patients and to perform early diagnoses. Last years, the neutrophil-to-lymphocyte ratio (NLR)

27 has been proposed as a simple biological parameter able to stratify the risk of mortality after a

28 major cardiac event and to predict cancer outcome. ${ }^{1-10}$

29 In this study, we investigated the potential correlation between the NLR, the C-Reactive Protein

30 (CRP) level and the postoperative complications following major abdominal surgery. 


\section{PeerJ Reviewing Manuscript}

\section{Patients and methods}

34 Patients

35 We performed a post-hoc analysis of previously collected data concerning 82 consecutive

36 patients (median age: 62 years, range: $27-80$, female/male: $32 / 50$ ) undergoing major abdominal

37 surgery included in a previous trial (NCT00816153). After ethical committee approval (CEBHF of

38 the Université catholique de Louvain, Chairperson: $\operatorname{Pr}$ J.-M. Maloteaux) and written informed consent, these patients were prospectively followed. We included adults (>18 years) without any major organ dysfunction, and scheduled for esophagectomy, gastric resection/suture,

41 hepatectomy, pancreatectomy, or intestinal and colorectal surgeries. ${ }^{11}$

42 Data collection

43 For each patient, a postoperative care team member recorded preoperative characteristics, the

44 NLR and CRP values (preoperative, at days $+1,+2$ and +7 ), and delayed postoperative

45 complications (between $D+8$ and $D+30$ ) such as infections, pulmonary embolism, acute

46 myocardial infarction, acute lung injury/acute respiratory distress syndrome, pulmonary edema,

47 arrhythmia, stroke, cardiac arrest, coagulopathy (platelets $<100,000 . \mathrm{\mu l}^{-1}$, international

48 normalized ratio $>2$ ), hepatic dysfunction, upper digestive hemorrhage, leakage of anastomosis,

49 and mortality.

50 Leukocytes count and CRP were typically included in the routine perioperative evaluation and

51 prospectively registered in a computed database. All venous blood samples were processed in a

52 blood analyzer (Sysmex [TOA Medical Electronics, Kobe, Japan]) for the determination of the

53 complete blood cell counts and differential counts of leukocytes. We recorded the neutrophils

54 and the lymphocytes counts, and calculated the neutrophil to lymphocyte ratio. ${ }^{2}$

55 The CRP was determined by turbidimetry (UniCel ${ }^{\circledR}$ DxC 800 [Beckman Coulter, Pasadena,

56 California, U.S.A.]) on a serum or plasma sample. During the reaction, a particle coated with

57 anti-CRP antibody binds to the CRP in the patient sample forming insoluble aggregates. The

58 system monitors the change in absorbance at 600 nanometers. This change in absorbance is

59 proportional to the concentration of CRP in the sample and is used by the system to calculate 
60 and express the concentration of CRP in a nonlinear calibration curve to a single point, adjusted

61 and predetermined. A value of $\mathrm{CRP}<1.0 \mathrm{mg} / \mathrm{dL}$ was considered as normal.

62 Statistical analysis

63 We compared patients with and without post-operative complications using Chi-square for

64 categorical variables and (paired) Student t-test for continuous ones as appropriate. Uni- and

65 multivariate analyses using logistic/linear regression models (with stepwise backward regression

66 for multivariate analysis) were used. $P<0.05$ was considered statistically significant. Data are

67 expressed as mean ( $\pm \mathrm{sd})$, mean [95\% confidence interval], or number (percentage).

68 STATISTICA (data analysis software system) version 7 (Statsoft, Inc., 2004) was used for all

69 analyses.

\section{Results}

72 Baseline characteristics, procedures and postoperative complications

73 Preoperative characteristics, type and duration of procedure are detailed in table 1.

74 During the postoperative period, 45 patients presented 69 complications. Two patients died from 75 septic shock 20 days and 33 days after surgery of a failed anastomosis (Table 2). Patients with 76 complications were comparable in term of preoperative characteristics, type and duration of 77 procedure $(P>0.05)$ (data not shown).

78 Inflammatory response after abdominal surgery

79 The NLR increased at D+1 and, on average, returned to baseline at D+7 unless complications

80 (Table 3, Figure) $(P<0.05)$. Preoperative NLR is not significantly associated with postoperative

81 complications whereas it is the case of NLR at D+7. CRP presents a delayed peak compared to

82 the NLR, increasing at D+2 and not normalizing at D+7, either there were complications or not 83 (Figure).

84 Univariate analysis

85 To detect potential confounders, we performed an univariate analysis using general 86 logistic/linear regression model to observe the possible associations between postoperative 
87 complications and preoperative variables. When investigating inflammatory markers, the NLR at

88 D+7 was associated with more complications $(P<0.0001)$ (Table 4$)$.

89 Multivariate analysis

90 When including these variables into a multivariate analysis, and using a general logistic/linear

91 regression model with stepwise backward regression, the NLR value at $D+7$ was the only

92 variable independently associated with more complications $(P<0.001)$, whereas none of the

93 other factors, including CRP, showed any independent correlation. To note that American Society

94 of Anesthesiologists Score (ASA) was not included because of high risk of colinearity of this

95 variable with other comorbidities, precluding its inclusion in a multivariate model. A performance

96 analysis showed an area-under-the receiver operating curve (AUC) of $68 \%$ [95\%Cl:56-81\%]

$97(P=0.006)$. The optimal cut-off of the NLR to predict occurrence of postoperative complications is

985.5 , with a sensitivity of $66 \%$ and a specificity of $77 \%$. The same analysis for the CRP did not

99 show any significant result $(P>0.05)$.

100

101 Discussion

102 Complications are associated with a greater inflammatory response to abdominal surgery. This

103 is better reflected by a significantly higher NLR, 7 days after the surgery, than by the CRP level.

104 Indeed, the NLR remains elevated in patients developing post-operative complications

105 compared with non-complicated outcome patients. In a multivariate analysis, the NLR value at

106 D+7 was the only factor associated with postoperative complications.

107 CRP levels have a delayed kinetics compared to the NLR, increasing at D+2 and not

108 normalizing at $D+7$ either there are complications or not.

109 Our results extend previous findings showing that NLR is an independent marker of impaired

110 outcome. In fact, the NLR has already been associated with morbidity or mortality of patients

111 with cancer, cardiovascular disease, or chronic renal failure. ${ }^{2,3,8,12,13}$ 
112 Specifically during the perioperative period, Vaughan-Shaw et al conducted a retrospective

113 analysis of patients aged 80 years or over undergoing emergency abdominal surgery.

114 Multivariate analysis identified NLR to be an independent predictor of 30-day outcome yet CRP

115 did not predict outcome at any time point. ${ }^{14}$

116 Taken together, this results show that NLR is a simple biomarker, widely available and probably

117 more efficient than CRP, that can be used in various type of populations to monitor the

118 inflammatory reaction and potentially able to help the clinician to perform early diagnoses of 119 postoperative complications.

120 As our work is not designed for, further works may focus on the influence of various factors on

121 the NLR, like the surgical technique (laparoscopy versus lapatomy) and anesthesia. Indeed,

122 some potentially interesting factors were not considered in this work. This is a limit of such kind

123 of post-hoc (retrospective) analysis. For example, the systemic IL-6 response is related to the

124 magnitude of surgical trauma, as reflected by the complexity of the surgical procedure and the

125 duration of the abdominal operation. ${ }^{15}$ Regarding the influence of the anaesthesia, Kim et al

126 demonstrated that TIVA with propofol and remifentanil compared with inhalational anesthesia

127 with sevoflurane could modify the leukocytic alterations, including neutrophil-to-lymphocyte ratio

128 in peripheral blood during the postoperative period of laparoscopy-assisted vaginal hysterectomy

129 while the significance of these changes remains largely unanswered. ${ }^{13,16}$ Finally, we have to

130 recognize that we did not prove any clinical usefulness, questionable for a marker available

131 seven days after surgery, knowing that earlier would be better. To note that CRP level, assessed

132 two days after surgery, was a potential earlier marker. Then, the possibility exist to consider both

133 CRP and NLR values in a predictive score. But, if statistically different in patients with

134 complications, CRP was not an independent predictor in multivariate analysis, challenging this 135 approach. 
Conclusion

137 In this series of patients, the NLR at D+7 is significantly associated with postoperative

138 complications after major abdominal surgery, and may be a simple but important biomarker as it 139 was not the case of CRP level.

140

141 Role of the authors: PF recruited the patients. PF and VD collected the data. PF performed the

142 analysis. PF, VD and MDK were involved in the design, the redaction of the manuscript and

143 accepted the final version.

144 Conflict of interest: None. 
146

147

148

149

150

151

152

153

154

155

156

157

158

159

160

161

162

163

164

165

166

167

168

169

170

171

172

173

174

175

176

177

178

179

180

181

182

183

184

185

186

187

188

189

190

191

192

193

194

195

1. Tabuchi T, Shimazaki J, Satani T, Nakachi T, Watanabe Y, Tabuchi T. The perioperative granulocyte/lymphocyte ratio is a clinical relevant marker of surgical stress in patients with colorectal cancer. Cytokine. 2011; 53(2):243-48.

2. Forget $P$, Machiels JP, Coulie PG, Berliere M, Poncelet AJ, Tombal B, Stainier A, Legrand C, Canon JL, Kremer Y, De Kock M. Neutrophil:Lymphocyte Ratio and intraoperative use of Ketorolac or Diclofenac are prognostic factors in different cohorts of patients undergoing breast, lung and kidney cancer surgery. Ann Surg Oncol. 2013 [Epub ahead of print]

3. Proctor MJ, Morrison DS, Talwar D, Balmer SM, Fletcher CD, O'Reilly DS, Foulis AK, Horgan PG, McMillan DC. A comparison of inflammation-based prognosis scores in patients with cancer. A Glasgow inflammation outcome study. Eur J Cancer. 2011; 47(17):2633-41.

4. Chua W, Charles KA, Baracos VE, Clarke SJ. Neutrophil/lymphocyte ratio predicts chemotherapy outcomes in patients with advanced colorectal cancer. $\mathrm{Br} J$ Cancer. 2011; 104(8):1288-95.

5. Keizman D, Ish-Shalom M, Huang $P$, Eisenberger MA, Pili R, Hammers $H$, Carducci MA. The association of pre-treatment neutrophil to lymphocyte ratio with response rate, progression free survival, and overall survival of patients treated with sunitinib for metastatic renal cell carcinoma. Eur J Cancer. 2012; 48(2):202-8.

6. Huang ZL, Luo J, Chen MS, Li JQ, Shi M. Blood neutrophil-to-lymphocyte ratio predicts survival in patients with unresectable hepatocellular carcinoma undergoing transarterial chemoembolization. J Vasc Interv Radiol. 2011; 22(5):702-9.

7. Kim HS, Han KH, Chung HH, Kim JW, Park NH, Song YS, Kang SB. Neutrophil to lymphocyte ratio for preoperative diagnosis of uterine sarcomas: a case-matched comparison. EurJ Surg Oncol. 2010; 36(7):691-8.

8. Ding PR, An X, Zhang RX, Fang YJ, Li LR, Chen G, Wu XJ, Lu ZH, Lin JZ, Kong LH, Wan DS, Pan ZZ. Elevated preoperative neutrophil to lymphocyte ratio predicts risk of recurrence following curative resection for stage IIA colon cancer. Int J Colorectal Dis. 2010; 25(12):1427-33.

9. Chiang SF, Hung HY, Tang R, Changchien CR, Chen JS, You YT, Chiang JM, Lin JR. Can neutrophil to lymphocyte ratio predict the survival of colorectal cancer patients who have received curative surgery electively ? Int J Colorectal Dis. 2012; 27(10):1347-57.

10.Sharaiha RZ, Halazun KJ, Mirza F, Port JL, Lee PC, Neugut Al, Altorki NK, Abrams JA. Elevated preoperative neutrophil:lymphocyte ratio as a predictor of postoperative disease recurrence in esophageal cancer. Ann Surg Oncol. 2011; 18(12):3362-9.

11. Forget $P$, Lois $F$, De Kock $M$. Goal-directed fluid management based on the pulse oximeter-derived pleth variability index reduces lactate levels and improves fluid management. Anesth Analg. 2010; 111(4):910-914.

12. Walsh SR, Cook EJ, Goulder F, Justin TA, Keeling NJ. Neutrophil-lymphocyte ratio as a prognostic factor in colorectal cancer. J Surg Oncol. 2005; 91(3):181-4.

13. Kim WH, Jin HS, Ko JS, Hahm TS, Lee SM, Cho HS, Kim MH. The effect of anesthetic techniques on neutrophil-to-lymphocyte ratio after laparoscopy-assisted vaginal hysterectomy. Acta Anaesth Taiwanica. 2011; 49(3):83-7.

14. Vaughan-Shaw PG, Rees JR, King AT. Neutrophil lymphocyte ratio in outcome prediction after emergency abdominal surgery in the elderly. Int J Surg. 2012; 10:15762.

15. Sido $B$, Teklote JR, Hartel $M$, Friess $H$, Büchler $M W$. Inflammatory response after abdominal Surgery. Best Pract Res Clin Anaesth. 2004; 18(3):439-54. 
16. Carli F, Annetta MG. Conséquences immunologiques de la chirurgie et de l'anesthésie: 198 mécanismes et modalités thérapeutiques. Prat Anesth Reanim. 2003 ; 7(4):231-47. 
1

Figure

A. C-reactive protein (CRP) and B. Neutrophil-to-Lymphocyte ratio (NLR) values in 82 patients: preoperatively, at day +1 (D1), day +2 (D2) and day +7 (D7), and presenting (or not) postoperative complications after major abdominal surgery. Data are presented as mean \pm SEM. P-value after (paired) Student t-tests. 


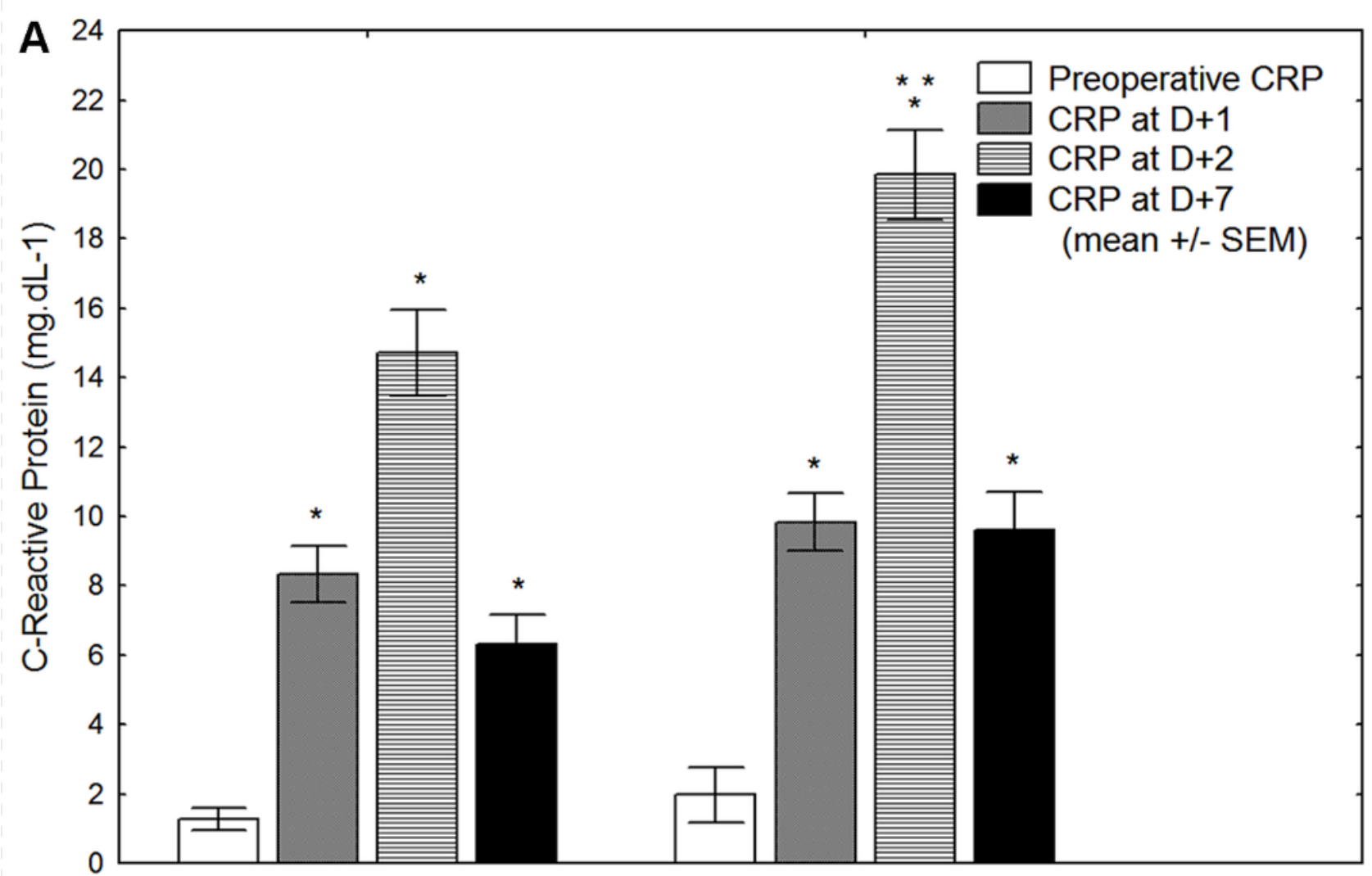

Patients without complication Patients with at least one complication $(\mathrm{N}=37)$ $(\mathrm{N}=45)$

${ }^{*} P<0.001$ compared with baseline ${ }^{* *} P<0.05$ comparing the groups

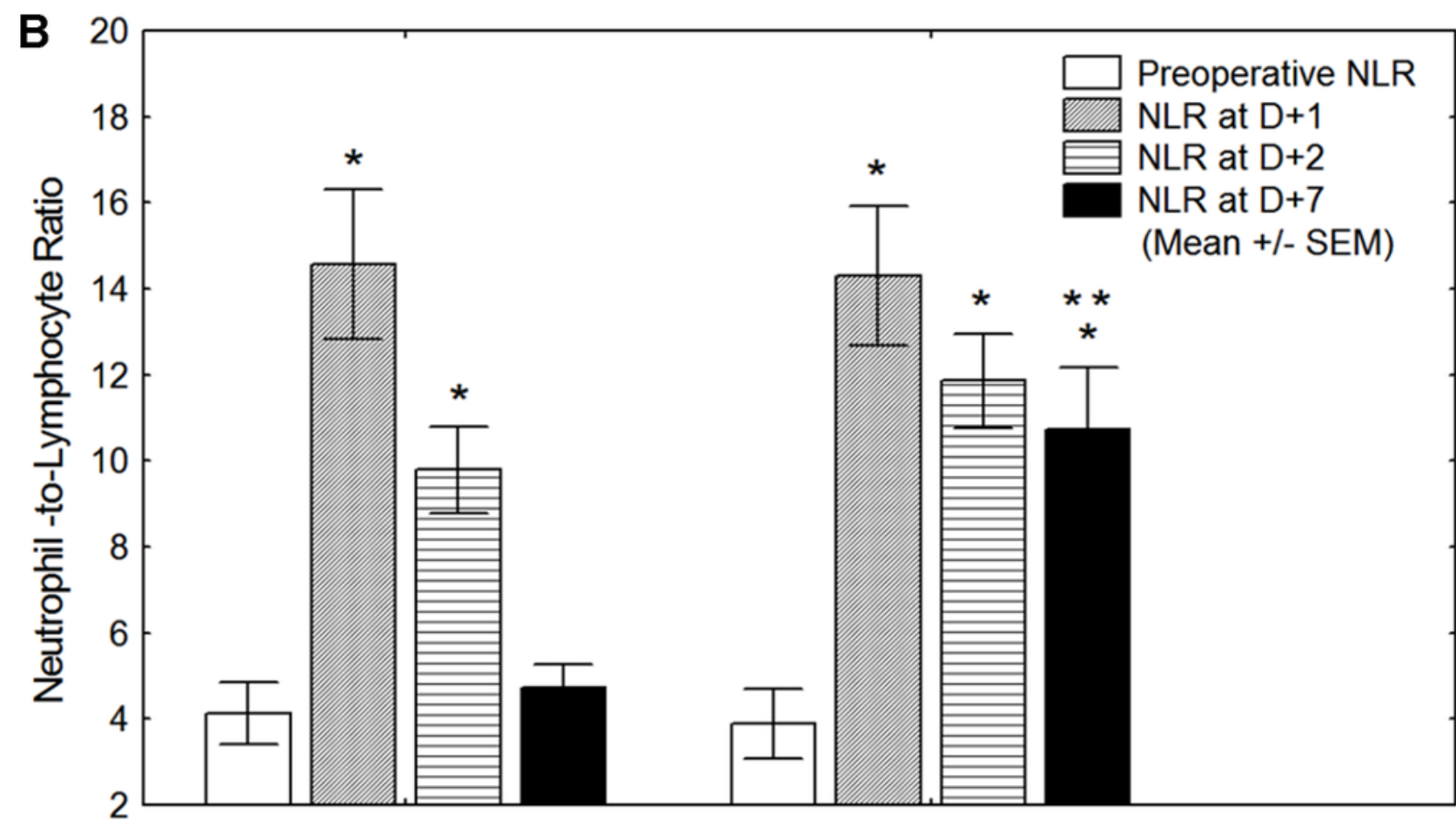

Patients without complication Patients with at least one complication $(\mathrm{N}=37)$ $(\mathrm{N}=45)$

* $P<0.001$ compared with baseline ${ }^{* *} P<0.001$ comparing the groups 


\section{Table $\mathbf{1}$ (on next page)}

Table 1

Preoperative Characteristics, Incidence of Chronic Diseases, Type and duration of Surgery and Anesthesia, Use of epidural analgesia. Data are represented as median (range), mean \pm SD or number (\%). Two patients had 2 types of surgery. 


\section{PeerJ Reviewing Manuscript}

Baseline characteristics

\begin{tabular}{ll}
\hline Age (years) & $62(27-80)$ \\
Weight $(\mathrm{kg})$ & $69.5 \pm 15.5$ \\
Height $(\mathrm{cm})$ & $169.5 \pm 9.0$ \\
Sex (female/male) & $32 / 50(39.0 / 60.9)$ \\
American Society of Anesthesiologists score $2 / 3$ & $44(53.6) / 38(46.3)$ \\
Chronic diseases & \\
$\quad$ Cirrhosis & $3(3.6)$ \\
$\quad$ Chronic Obstructive Pulmonary Disease & $4(4.8)$ \\
$\quad$ Hypertension & $31(37.8)$ \\
$\quad$ Peripheral vascular disease & $14(17.0)$ \\
$\quad$ Coronary artery disease & $7(8.5)$ \\
$\quad$ Other cardiomyopathy & $6(7.3)$ \\
$\quad$ Diabetes mellitus & $6(7.3)$ \\
Preoperative biological values & \\
$\quad$ Hemoglobin (g.dl- ${ }^{-1}$ ) & $12.6 \pm 2.0$ \\
$\quad$ Serum creatinine (mg.dl & \\
Type of surgery & $0.96 \pm 0.3$ \\
$\quad$ Upper gastrointestinal & $12(14.6)$ \\
$\quad$ Hepato-biliary & $26(31.7)$ \\
$\quad$ Lower gastrointestinal & $46(56.1)$ \\
Laparoscopic approach & $10(12.2)$ \\
Duration of surgery (minutes) & $298 \pm 139.5$ \\
Duration of anesthesia (minutes) & $351 \pm 141.5$ \\
Epidural analgesia & $62(75.6)$
\end{tabular}




\section{Table 2 (on next page)}

Table 2

Postoperative outcome: Complications, events and intensive care unit/hospital stay. Data are represented as mean \pm SD or number (\%).Other infections than of surgery site concern: pulmonary, line-related, and other abdominal infection (like urologic infections).

Cardiovascular complications concern: acute myocardial infarction, acute lung injury / acute respiratory distress syndrome, pulmonary edema, or arrhythmia. 


\section{PeerJ Reviewing Manuscript}

Postoperative outcome

Postoperative complications (N)

Infection of surgery site

Other infections

Cardiovascular complications

Coagulopathy

Upper digestive hemorrhage

Leakage of anastomosis

Morbidity (event per patient)

Mortality

Length of stay

Postoperative mechanical ventilation Intensive care unit (days)

Hospital (days)
69

$16(19.5)$

$13(15.8)$

$12(14.6)$

$11(13.4)$

$7(8.5)$

$10(12.2)$

$1.35 \pm 2.0$

$2.0(2.4)$

4 (4.9)

$2.0 \pm 6.5$

$15.6 \pm 16.1$ 


\section{Table 3 (on next page)}

Table 3

C-reactive protein (CRP) and Neutrophil-to-Lymphocyte ratio (NLR) values in 82 patients: preoperatively (preop), at day +1 (D1), day +2 (D2) and day +7 (D7), and presenting (or not) postoperative complications after major abdominal surgery. Data are presented as mean \pm SD. $P$ value $<0.05$ is considered significant. $* P<0.05$ and $* * P<0.001$ comparing the patients with and without complications (Student t-test). $\uparrow \mathrm{P}<0.001$ compared with preoperative value (paired Student t-test). 


\section{PeerJ Reviewing Manuscript}

\begin{tabular}{cccc} 
& $\begin{array}{c}\text { Total } \\
(\mathrm{N}=82)\end{array}$ & $\begin{array}{c}\text { With complications } \\
(\mathrm{N}=45)\end{array}$ & $\begin{array}{c}\text { Without complication } \\
(\mathrm{N}=37)\end{array}$ \\
\hline CRP preop & $1.67 \pm 3.74$ & $1.98 \pm 4.80$ & $1.29 \pm 1.76$ \\
CRP D1 & $9.17 \pm 5.44$ & $9.84 \pm 5.71 \dagger$ & $8.34 \pm 5.06 \dagger$ \\
& & & \\
CRP D2 & $17.56 \pm 8.89$ & $19.84 \pm 9.06 \dagger$ & $14.72 \pm 7.91^{*} \dagger$ \\
& & & \\
CRP D7 & $8.13 \pm 6.88$ & $9.60 \pm 7.74 \dagger$ & $6.32 \pm 5.20 \dagger$ \\
\hline NLR preop & $4.0 \pm 4.91$ & $3.89 \pm 5.35$ & $4.13 \pm 4.43$ \\
NLR D1 & $14.43 \pm 11.12$ & $14.30 \pm 11.37 \dagger$ & $14.58 \pm 10.97 \dagger$ \\
NLR D2 & $10.98 \pm 7.07$ & $11.87 \pm 7.64 \dagger$ & $9.79 \pm 6.13 \dagger$ \\
NLR D7 & $7.96 \pm 8.12$ & $10.73 \pm 9.86 \dagger$ & $4.73 \pm 3.38{ }^{* *}$
\end{tabular}




\section{Table 4 (on next page)}

Table 4

Uni- and multivariate analysis regarding risk factors of postoperative complications in 82 patients undergoing major abdominal surgery. C-reactive protein (CRP) and Neutrophil-toLymphocyte ratio (NLR) values are considered at day+1 (D1), day +2 (D2) and day +7 (D7). 
PeerJ Reviewing Manuscript

\begin{tabular}{lcccc} 
& $\begin{array}{c}\text { Odds } \\
\text { Ratio }\end{array}$ & $\begin{array}{c}\mathbf{9 5 \%} \text { Confidence } \\
\text { Interval }\end{array}$ & $\boldsymbol{P}$ \\
\hline Univariate analysis & \multicolumn{5}{c}{. } & & \\
\hline Cirrhosis & 1.66 & 1.08 & 2.56 & 0.02 \\
Peripheral vascular disease & 1.65 & 1.33 & 2.04 & $<0.001$ \\
Coronaropathy & 1.62 & 1.23 & 2.14 & $<0.001$ \\
CRP preop & 1.03 & 0.90 & 1.18 & 0.64 \\
CRP D1 & 0.96 & 0.90 & 1.03 & 0.27 \\
CRP D2 & 1.03 & 0.99 & 1.06 & 0.10 \\
CRP D7 & 0.97 & 0.93 & 1.01 & 0.15 \\
NLR preop & 0.97 & 0.87 & 1.08 & 0.59 \\
NLR D1 & 0.98 & 0.95 & 1.01 & 0.18 \\
NLR D2 & 1.02 & 0.97 & 1.07 & 0.37 \\
NLR D7 & 1.05 & 1.01 & 1.09 & 0.01 \\
\hline Multivariate analysis & & & & \\
\hline NLR D7 & 1.03 & 1.01 & 1.04 & $<0.001$
\end{tabular}

